\title{
VII. Auflösung des Instituts 99
}

Mit dem Kriegsende verlor das Institut 99 einen wesentlichen Teil seiner Aufgaben. Standen im Frühsommer 1945 noch die Kadereinsätze in Deutschland im Mittelpunkt der Tätigkeit, so wurde zum Jahresende die Parteischulung eingestellt. Ebenfalls zum Ende des Jahres 1945 erfolgte die Auflösung des Nationalkomitees „Freies Deutschland“, das seinerzeit der Impuls für die Gründung des Instituts 99 gewesen war. Ab 1946 wurde in den Lagern die politische Erziehung unter den Kriegsgefangenen wieder unter der alten Bezeichnung „antifaschistische Bewegung“ (Antifa) fortgesetzt. Die Antifa-Schulung lief weiter, änderte im Laufe des Jahres 1946 aber ihr Profil. Damit einher ging die Diskussion um die Verwendung der Antifa-Schüler als Kader in Deutschland. Schließlich wurden die Antifa-Schulen Anfang 1947 umstrukturiert. Das Ende des Nationalkomitees war der eine, die Profiländerung der Antifa-Schulung der andere Schritt, die beide zusammen zur Auflösung des Instituts 99 führten.

\section{Das Ende des Nationalkomitees}

Anfang Juni 1945 begann im Institut 99 die Diskussion um die zukünftige Tätigkeit von Zeitung und Sender „Freies Deutschland“. Die Radioredaktion „Freies Deutschland“ hielt eine Veränderung des Profils für notwendig, „um das Nationalkomitee nicht zu diskreditieren“. Künftig solle über das Leben in der Sowjetunion und in der SBZ berichtet werden. Aus dem Lager des NKFD in Lunevo sollten Vorträge über die Umerziehung des deutschen Volkes übertragen sowie Grüße und Berichte deutscher Soldaten aus den Lagern in die Heimat gesendet werden ${ }^{1}$. Auch der Leiter der Zeitungsredaktion, Lothar Bolz, wies auf die vollkommen veränderte Aufgabe der Zeitung hin. Sie solle nicht mehr zur „Zersetzung der deutschen Fronttruppen" beitragen, sondern habe „bei der Umerziehung und Schulung der deutschen Kriegsgefangenen im Geiste des Antifaschismus zu helfen und ihre Arbeitsproduktivität zu steigern". Diese neue Zielstellung erfordere eine personelle Aufstockung der Redaktion - etliche Mitarbeiter waren nach Deutschland zurückgekehrt - und eine direkte Unterstellung unter das UPVI. Denn die Zeitungsredaktion müsse endlich Verbindung mit allen Kriegsgefangenenlagern bekommen, die bislang nicht bestanden habe: „Da das Material, dessen die Redaktion bedarf, zum Teil streng vertraulichen Charakter hat und das Volkskommissariat für innere Angelegenheiten einer ihr nicht unmittelbar unterstellten Organisation solches Material nicht zugänglich machen wird und sie auch nicht über seine laufenden Maßnah-

1 „Defizite der derzeitigen Sendungen“, SAPMO-BArch NY 4065/18, Bl. 11; vgl. das dem Papier zugrundeliegende Konzept, ebenda Bl. 1-4. 
men unterrichten kann, erscheint es als zweckmäßigste Lösung, die Redaktion derselben Behörde zu unterstellen, der die Kriegsgefangenenlager unterstehen, das heißt: der Verwaltung der Kriegsgefangenenlager beim Volkskommissariat für Innere Angelegenheiten. "2 Die Vorschläge der Redaktionen wurden von NKFDPräsident Weinert an Burcev, den Leiter der 7. Verwaltung weitergereicht. Allerdings schwächte Weinert die Forderung nach strukturellen Veränderungen $a b$, indem er immer noch von einer „Fortsetzung der publizistischen Tätigkeit des Nationalkomitees Freies Deutschland" sprach. Eingedenk des Funktionierens sowjetischer Verwaltungsstrukturen präzisierte aber auch er: „Unter Fortsetzung der Arbeit der Zeitungs- und Radioredaktion verstehe ich nicht, daß sie nur existieren, sondern daß die beiden Organe auch nach Beendigung des Krieges die vor ihnen stehenden und politisch wichtigen Aufgaben erfüllen sollen. Diese Aufgaben müssen politisch und technisch einwandfrei ausgeführt werden. "3

Gegenüber dem OMI wurde die Frage nach dem Fortbestand des Nationalkomitees erst am 9. Juli 1945 angesprochen. Offensichtlich hatte die 7. Verwaltung die Gründung „antifaschistisch-demokratischer" Parteien in der SBZ abgewartet - als letzte der vier neuen Parteien wurde die Liberaldemokratische Partei Deutschlands am 5. Juli gegründet -, bevor sie nun die Auflösung des NKFD vorschlug ${ }^{4}$. Burcev und Braginskij begründeten dies mit der Zusammenarbeit der Parteien in der „Einheitsfront der antifaschistisch-demokratischen Parteien". Bevor der Block der Parteien in der SBZ an die Öffentlichkeit treten werde - was am 15. Juli 1945 geschah solle sich das Nationalkomitee auflösen. Der Parteienblock könne dann - so die Absicht der 7. Verwaltung - öffentlich das Erbe des Nationalkomitees antreten. Dem OMI wurde vorgeschlagen: „Da in Deutschland der demokratische Block [der Parteien] seine Tätigkeit aufgenommen hat, scheint es entsprechend der bereits vorliegenden Meinungen [Weinerts] in dieser Angelegenheit zweckmäßig zu sein, die Frage nach der Liquidierung des Nationalkomitees ,Freies Deutschland' zu stellen. Am 12. Juli ist der zweite Jahrestag der Gründung des Komitees. Möglicherweise wäre es zweckmäßig, zu diesem Tag eine öffentliche Erklärung des Nationalkomitees herauszugeben, daß es seine Tätigkeit einstelle, weil im Zusammenhang mit der Gründung des demokratischen Blockes in Deutschland die Aufgaben der Bewegung Freies Deutschland als erfüllt angesehen werden können. Entsprechend dieser Verlautbarung könnten die Radiosendungen eingestellt und die Zeitung ,Freies Deutschland' umgewandelt werden in eine Zeitung für deutsche Kriegsgefangene in der Sowjetunion mit dem Namen, Wöchentliche Rundschau“" 5 Die 7. Verwaltung forcierte Weinerts Vorschläge. Sie bezog sich aber in ihrer Argumentation nicht auf die politische Erziehung in den sowjetischen Kriegsgefangenenlagern, sondern auf die aktuelle Entwicklung in der SBZ. Denn die 7. Verwaltung war in Deutschland intensiv mit dem Aufbau antifaschistischer Selbstverwaltungen, Zeitungen und Radiostationen beschäftigt, worüber sie Anfang Juli in Moskau berichtet hatte. Es war daher nur konsequent, die Auflösung des NKFD in diesen Kontext zu stellen, zumal es abschließend in dem Bericht hieß: „Die Wiedergrün-

2 Bolz an Weinert am 6. 6. 1945, ebenda, Bl. 7.

3 Weinert an Burcev am 10. 6. 1945, ebenda, Bl. 12.

4 Zur Entwicklung der Parteien in der SBZ siehe Weber, Geschichte der DDR, S. $69 \mathrm{ff}$.

5 Burcev und Braginskij an Dimitrov am 9. 7. 1945, RGASPI 17/128/839, Bl. 124. 
dung der Kommunistischen Partei Deutschlands und die bevorstehende Bildung der anderen antifaschistischen Parteien und der freien Gewerkschaften mit ihrer Presse erweitern die Möglichkeiten zur Beeinflussung der deutschen Bevölkerung in beträchtlichem Maße." 6 Die Frage der Fortsetzung der politischen Arbeit unter den Kriegsgefangenen in der Sowjetunion und der damit eventuell notwendigen strukturellen Veränderungen von Institut $99 \mathrm{bzw}$. UPVI wurden ausgeklammert. Im weiteren Verlauf der sowjetischen Entscheidungsfindung über die Auflösung des NKFD standen deutschlandpolitische Bezüge im Vordergrund.

Im Juli 1945 kam es nicht zur Auflösung des Nationalkomitees. Da aber der fortgesetzte Sendebetrieb des NKFD zunehmend störte ${ }^{7}$ - in Deutschland arbeiteten inzwischen mehrere „antifaschistische“ Stationen -, stellte das Institut 99 den Betrieb des Senders „Freies Deutschland“ am 8. September 1945 „schlagartig“ ein 8 . Dadurch verlor das Nationalkomitee nicht nur seine Außenwirkung, sondern faktisch jegliche Wirkung, denn die Zeitung „Freies Deutschland“ war nur einem sehr kleinen Kreis von Lesern in den Kriegsgefangenenlagern zugänglich. Im Grunde war damit die Auflösung des Nationalkomitees vollzogen. Es erstaunt daher auch nicht, daß sich der Chef des GlavPURKKA bei Weinert erkundigte, "ob es noch irgendeinen Beweggrund für den Fortbestand des Nationalkomitees gebe "9. Vom GlavPURKKA zu einer Stellungnahme aufgefordert, wandte sich Weinert an Dimitrov, wobei er sich die Argumentation der Politischen Hauptverwaltung zu eigen machte: Das Nationalkomitee habe angesichts der "Schaffung einer breiten antifaschistischen Front in Deutschland" seine Aufgabe erfüllt. Der Sender könne seine Tätigkeit einstellen, die Zeitung jedoch solle in ein „zentrales Organ der Kriegsgefangenen" umgewandelt werden. Weinert blieb angesichts der vollendeten Tatsachen wie dem bereits existierenden Parteienblock in der SBZ sowie dem ohnehin schon eingestellten Sendebetrieb des NKFD als einziges Argument der außenpolitische Effekt der Komiteeauflösung: „Ich halte es angesichts der Tatsache, daß die Gründung des Nationalkomitees im Juli 1943 [in der sowjetischen Presse] veröffentlicht worden ist, für unabdingbar, den Beschluß des Nationalkomitees über seine Selbstauflösung besonders in den in Deutschland erscheinenden Zeitungen mit einer kurzen Erklärung zu publizieren, insbesondere deshalb, weil sich zahlreiche antifaschistische deutsche Gruppen als Filialen des Nationalkomitees betrachten." 10 Weinerts Vorschläge wurden am 21. September von Dimitrov an Stalin geschickt mit dem Vorschlag, einen „begründeten“ Beschluß des Nationalkomitees über seine Selbstauflösung herbeizuführen. Die Zeitung "Freies Deutschland“ solle in eine Zeitung für deutsche Kriegsgefangene umgewandelt werden und - darum hatte Weinert gebeten - der privilegierte Status der ehemaligen NKFD-Mitglieder erhalten bleiben, um die loyal zur Sowjetunion stehenden Aktivisten später zum Einsatz

6 „Auskunft der Politischen Hauptverwaltung der Streitkräfte der UdSSR über die politische Arbeit unter der Bevölkerung Deutschlands" vom 5. 7. 1945, in: Sowjetische Politik in der SBZ, S. 10.

7 Vgl. Ulbricht an Dimitrov am 9. 5. 1945, in: „Gruppe Ulbricht“, S. 323: „Die Propaganda des Senders ,Freies Deutschland' führt ebenfalls in die Irre, da wir die Örtlichen Komitees ,Freies Deutschland' liquidieren.".

8 Diesener, Propagandaarbeit, S. 165.

9 Darüber berichtete Weinert am 19.9. 1945 an Dimtrov, RGASPI 17/128/839, Bl. 138.

10 Ebenda, Bl. 139-140. 
nach Deutschland zu schicken bzw. für die Arbeit unter den deutschen Kriegsgefangenen zu nutzen ${ }^{11}$.

Bevor die Erlaubnis zur Auflösung des NKFD erging, wurde das Einverständnis des NKVD eingeholt. Berija erklärte seine Zustimmung gegenüber Stalin am 30. September mit der lakonischen Begründung, der Bedarf an NKFD und BDO, die in der letzten Zeit ohnehin nicht gearbeitet hätten, sei entfallen ${ }^{12}$. Einen identischen Brief schickte Berija auch an Molotov, der am 5. Oktober seine Einwilligung gab $^{13}$. Schließlich überreichte Dimitrov am 5. Oktober Bulganin „den Brief an Genosse Stalin (inklusive Anlagen) zu der Frage des Nationalkomitees ,Freies Deutschland", entsprechend unserer Absprachen"14. Die endgültige Entscheidung über die Auflösung des NKFD fiel höchstwahrscheinlich am Abend des 5. Oktober, als sich Molotov, Bulganin und zeitweilig auch Berija bei Stalin einfanden ${ }^{15}$. Allerdings hatte es mit der Umsetzung der Entscheidung keine Eile. Der entsprechende NKVD-Befehl erging erst am 2. November 1945, an dem Tag, an dem das Nationalkomitee seine Selbstauflösung beschloß16. Offiziell wurde das Ende des Nationalkomitees damit begründet, daß - wie seit Juli 1945 immer wieder als Argument vorgebracht - „nach der völligen Vernichtung des Hitlerstaates und nach der Entfaltung der Tätigkeit des demokratischen Blocks der antifaschistischen Parteien in Deutschland" die weitere Tätigkeit von NKFD und BDO entfalle ${ }^{17}$. Für die sowjetische Deutschlandpolitik ist diese Begründung nicht mehr nutzbar gemacht worden. Der Block antifaschistisch-demokratischer Parteien berief sich nie auf das NKFD ${ }^{18}$.

11 Dimitrov an Stalin am 21. 9. 1945, RGASPI 17/128/716, Bl. 107-108. Die als Anlage beigefügten Vorschläge Weinerts befinden sich nicht in der Akte.

12 Berija an Stalin am 30. 9. 1945, GARF 9401/2/99, Bl. 399.

13 Berija an Molotov am 30. 9. 1945, GARF 9401/2/104, Bl. 2; ebenda handschriftlicher Zusatz „Ich bin einverstanden, V. Molotov, 5. 10. 45"; Edition des Briefes in: SSSR i germanskij vopros, tom II, S. 245.

14 Dimitrov an Bulganin am 5. 10. 1945, RGASPI 17/128/839, Bl. 136. Bulganin war Mitglied des Politbüros und des Staatlichen Verteidigungskomitees, außerdem stellvertretender Volkskommissar für Verteidigung und damit formal Stalins Stellvertreter.

15 Posetiteli kremlevskogo kabinteta, S. 112. Siehe demgegenüber Reschin (Feldmarschall im Kreuzverhör, S. 187), der die Absprachen zwischen OMI und operativer Abteilung des UPVI zur Auflösung des NKFD auf den 26.10. 1945 und den endgültigen Beschluß des Politbüros auf den 30.10. 1945 datiert. Das Besucherbuch Stalins weist aus, daß Stalin zu dieser Zeit im Urlaub war, so daß die Abstimmung mit ihm vermutlich schon Anfang Olktober erfolgte.

16 Handschriftlicher Vermerk des Sekretariats des NKVD auf dem Schreiben Berijas an Molotov vom 30. 9. 1945 mit dessen Zustimmung vom 5. 10. 1945 „NKVD-Befehl Nr. 0268 vom 2. 11. 45 ergangen", GARF 9401/2/104, Bl. 2. Der Brief ging, nachdem er von Molotov abgezeichnet worden war, wieder zurück an das NKVD; siehe ebenda Eingangsstempel des NKVD vom 3.11. 1945.

17 „Beschluß des Nationalkomitees Freies Deutschland“, in: FD Nr. 44/1945 vom 3. 11. 1945, S. 1.

18 Vgl. Benser, Zur Auflösung des Nationalkomitees, S. 906. Ebenda, S. 914 versucht Benser dennoch, eine ideelle Verbindung herzustellen: „Wenn es auch den direkten politisch-organisatorischen Brückenschlag vom NKFD zum politischen Neuansatz nach der Befreiung vom Faschismus nicht gegeben hat, so war dennoch die Bewegung ,Freies Deutschland' geschichtsträchtig nicht allein im allgemeinen Sinne dieses Wortes, sondern auch im Sinne einer vielfältigen, nachweisbaren Einflußnahme auf den Prozeß der antifaschistisch-demokratischen Umgestaltung auf deutschem Boden." 
Die Auflösung des Nationalkomitees war allein deswegen von Bedeutung, weil das NKFD seinerzeit mit großer Aufmerksamkeit bedacht worden war und nun nicht einfach verschwinden konnte. Sie war wichtig im Hinblick auf das sich verschlechternde Verhältnis zu den Westalliierten, um den Gerüchten über eine zukünftige Funktion der NKFD-Mitglieder in der SBZ oder gar einer versteckten Aufrüstung („Paulus-Armee“) keine Nahrung zu geben ${ }^{19}$. Leider geben die vorhandenen Quellen keine weiteren Einblicke in die sowjetische Motivation zur Auflösung des NKFD. Zumindest ist es nicht ausgeschlossen, daß gerade die argwöhnische Haltung der Westalliierten dazu führte, das NKFD nicht in die Tätigkeit des Blockes antifaschistischer Parteien in der SBZ münden zu lassen.

Nach der Auflösung des Nationalkomitees übernahm das UPVI die alleinige Regie bei der politischen Arbeit in den Kriegsgefangenenlagern. Es griff dabei auf das Erziehungskonzept zurück, das schon vor der Gründung des Nationalkomitees unter der Bezeichnung "Antifaschistische Bewegung" etabliert worden war. Ausgehend von dem Bestreben, Kriegsgefangene zu "Freunden der Sowjetunion" zu erziehen, wurde Antifaschismus nun mit der „Pflicht zur Wiedergutmachung“, d.h. dem Arbeitseinsatz in der Sowjetunion, gleichgesetzt. Die Zeitung „Freies Deutschland" stellte ihr Erscheinen ein, und die ab Januar 1946 erscheinende Kriegsgefangenenzeitung "Nachrichten“ erklärte die Wiedergutmachung zur Hauptaufgabe der Kriegsgefangenen. Der Schwerpunkt der politischen Arbeit unter den Kriegsgefangenen verlagerte sich auf die Aktivs in den Lagern, auf die das NKFD ohnehin keinen Einfluß bekommen hatte. Laut Robel begann damit die dritte Phase der Antifa, deren erste Phase (Sommer 1941 bis Sommer 1943) lediglich durch die Gründung des NKFD - die zweite Phase der Antifa - unterbrochen worden war: „Nach der Auflösung des NKFD und BDO tat sich der Wandel, der sich vollzogen hatte, auch nach außen hin kund: An ihre Stelle trat die ,antifaschistische Bewegung', kurz ,Antifa' genannt. Dieser Rückgriff auf eine Bezeichnung, unter der bis zur Gründung des NKFD die politische Arbeit in den Kriegsgefangenenlagern firmiert hatte, war programmatisch, und den ,Antifaschistischen Aktivs' der Lager, die auch in der Zeit des NKFD weiterbestanden hatten, wurde nun die alleinige Umerziehungsarbeit übertragen. " 20

Die Rückbesinnung auf die Antifa hatte eine Umstellung der politischen Arbeit in den einzelnen Kriegsgefangenenlagern zur Folge. Robel folgerte daraus, die Kriegsgefangenen seien erst 1946 von der Politischen Hauptverwaltung an das UPVI überstellt worden ${ }^{21}$. Diese Beobachtung ist nur insofern zutreffend, als sich das GlavPURKKA nach Auflösung des Nationalkomitees vollkommen von der politischen Arbeit mit den Kriegsgefangenen zurückzog. Das Institut 99 aber wurde nicht aufgelöst, sondern hatte nach wie vor die Aufgabe, die politische Arbeit unter den Kriegsgefangenen zu organisieren. Mit Auflösung des Nationalkomitees wechselte zwar die Institutsleitung, Kozlov wurde durch Romanov abgelöst, und die

$19 \mathrm{Zu}$ den Wahrnehmungen des NKFD durch die Briten, Franzosen und Amerikaner siehe Bungert, Das Nationalkomitee und der Westen, S. $257 \mathrm{ff}$.

20 Robel, Antifa, S. 95; vgl. ebenda S. 15 ff., 59 ff. und $165 \mathrm{ff}$.

21 Ebenda, S. 101. 
Abteilung für das Nationalkomitee fiel weg. Alle anderen Aufgaben aber - AntifaSchulen, Redaktionen, Lagerbevollmächtigte, Kaderregistrierung - blieben wie bisher bestehen ${ }^{22}$.

Struktur des Instituts $99(1946)^{23}$

\author{
Institutsleitung \\ Verlagsabteilung \\ Kaderabteilung \\ geheime Abteilung \\ Wirtschaftsabteilung \\ Buchhaltung
}

Antifa-Schule Krasnogorsk

deutscher Sektor

österreichischer Sektor

rumänischer Sektor

ungarischer Sektor

Antifa-Schule Juža

deutscher Sektor

österreichischer Sektor
Bevollmächtigte für die Arbeit in den Kriegsgefangenenlagern

Registratur kriegsgefangener Antifaschisten
Kriegsgefangenenzeitungen deutsche Redaktion österreichische Redaktion rumänische Redaktion ungarische Redaktion italienische Redaktion

Für die organisatorischen Strukturen der politischen Arbeit unter den Kriegsgefangenen blieb die Auflösung des NKFD ohne Konsequenzen. Dabei hatte der Chefredakteur der Zeitung „Freies Deutschland“, Lothar Bolz die Notwendigkeit insbesondere einer Strukturveränderung angesprochen, sollte die Wirkung der Kriegsgefangenenzeitung nicht verpuffen. Gemeinsam mit dem NKFD-Präsidenten Weinert und dem Leiter des Auslandsbüros der KPD, Försterling, hatte er „im Hinblick darauf, daß das Nationalkomitee in naher Frist in der SU nicht mehr bestehen dürfte", einen kompletten Plan zu Reorganisation der Antifa ausgearbeitet ${ }^{24}$. Dieser Plan wurde in einer leicht überarbeiteten Fassung am 17. August 1945 dem OMI mit der Erläuterung übergeben: „Die gegenwärtige Stagnation der politischen Arbeit unter den Kriegsgefangenen ist wohl als ein Übergangsstadium zu betrachten, das möglichst bald überwunden werden muß." 25 In dem Papier wurde grundsätzlich zu der Fortführung der politischen Arbeit unter den Kriegsgefangenen Stellung genommen: Nach dem Ende des Krieges bestünden die hauptsächlichen Aufgaben in den Kriegsgefangenenlagern in der Wiedergutmachung durch den Arbeitseinsatz von deutschen Kriegsgefangenen und in deren Umerziehung, damit

22 Das Protokoll über die Auflösung des NKFD am 2.11. 1945 vermerkt noch die Anwesenheit Kozlovs; Verrat hinter Stacheldraht?, S. 254.

23 Schema des Instituts 99, RGASPI 17/128/1150, Bl. 4.

24 „Plan der Organisierung der Arbeit unter den deutschen Kriegsgefangenen“ von Weinert, Försterling und Bolz, ohne Datum (vermutlich Juni/Juli 1945), SAPMO-BArch NY 4065/12, Bl. 12-13.

25 Försterling an Dimitrov am 17. 8. 1945, SAPMO-BArch DY 30/IV 2/11/197, Bl. 7. Es handelt sich um eine Abschrift, auf der der Adressat nicht genannt ist. Aber aufgrund aller bisher dargestellten strukturellen Zusammenhänge des sowjetischen ZK-Apparates kann es sich nur um Dimitrov bzw. das OMI handeln. 
sie als „aktive Kämpfer für eine demokratische Umgestaltung Deutschlands“ in ihre Heimat zurückkehren könnten. Um diese Ziele erreichen zu können, sei die „ständige unmittelbare operative Zusammenarbeit" von NKVD, GlavPURKKA und den einzelnen Lagerleitungen notwendig, die aber, wie die Erfahrung des Nationalkomitees gezeigt habe, „bei der bisherigen Organisationsstruktur" nicht zustande gekommen sei. Försterling schlug daher vor, aus Vertretern der KPD, des NKVD und des GlavPURKKA eine "zentrale Leitung" zu schaffen, die dem ZK der VKP (b) unmittelbar zu unterstellen sei. Es solle eine operative („sekrete“) Abteilung, eine Abteilung für Agitation und Propaganda und eine Schulungsabteilung eingerichtet werden. Ferner solle von der Zentrale eine Kriegsgefangenenzeitung herausgeben, ein Sender für Kriegsgefangene betrieben und Informationen für die Politinstrukteure erstellt werden. In den Lagern selbst solle ein der Zentrale verantwortlicher Lagerbevollmächtigter eine Lagergruppe („Aktiv“) leiten. Regelmäßige Delegationsreisen von der Zentrale aus sollten außerdem eine Überprüfung der Arbeit in den Lagern ermöglichen ${ }^{26}$.

Mit ihren Vorschlägen zur Reorganisation der politischen Arbeit unter den Kriegsgefangenen sprach die KPD den "Geburtsfehler" des Instituts 99 an. Bedingt durch die Rivalitäten zwischen GlavPURKKA und NKVD hatte das Institut die ihm zugedachte Koordinierungsfunktion immer nur in sehr eingeschränktem Maße wahrnehmen können. Auch die Gründung des OMI, und die damit gewährleistete Anbindung an das ZK der VKP (b), hatten das Institut 99 nicht aufwerten können. Die Interessen aller beteiligten Institutionen, die am deutlichsten bei der Verwendung der Kriegsgefangenen als Kader zutage traten, waren zu unterschiedlich. So scheute das Institut 99 bei der Auswahl der Schüler für die Schule Nr. 12 im Herbst 1944 den Konflikt mit dem UPVI. Ebenso wurden bei den Kaderplanungen für die Einsätze in Deutschland die Kriegsgefangenen an den Fronten der Roten Armee vom OMI nicht einbezogen. Da aber die KPD - wenn überhaupt nur über die Mitarbeit im Nationalkomitee Einfluß auf die Kaderrekrutierung nehmen konnte, befürchtete sie mit der Auflösung des Nationalkomitees den kompletten Verlust der Kaderbasis durch die Antifa-Schulen. Diese Sorge teilte Weinert am 3. November, einen Tag nach der Auflösung des NKFD, Wilhelm Pieck in Berlin mit: „Unter den Schülern ist eine große Anzahl sehr entwickelter und brauchbarer Leute. Ich habe aber festgestellt, daß das politische Kapital, das sie für uns darstellen könnten, schlecht angelegt wird. Wenn die Absolventen in die Reserve kommen und Holz hacken oder als ,Aktivisten' in Arbeitslager, wo sie weder weiterstudieren noch politische Arbeit leisten können, so geht alles wieder verloren, was mit Mühe erreicht worden war. Aber trotz aller Beschwerden darüber, die ich sogar persönlich beim obersten Chef [des UPVI] vorgebracht habe, hat sich bisher nichts geändert. Ähnlich liegen die Dinge mit den Kommunisten unter den Kriegsgefangenen." 27

Nach der Auflösung des Nationalkomitees und der Rückbesinnung auf die Antifa überschnitten sich ab Januar 1946 die Zuständigkeiten von UPVI und Institut 99 zunehmend. Das störte vermutlich solange nicht, wie der Aufbau der Antifa-

26 Ebenda, Bl. 8-9.

27 Weinert an Pieck am 3. 11. 1945, SAPMO-BArch NY 4065/5, Bl. 41-43. 
Aktivs in den Lagern noch nicht abgeschlossen war ${ }^{28}$. Aber wegen des wachsenden Bedarfs des UPVI an antifaschistisch geschulten Kriegsgefangenen eskalierte der Streit um die Verwendung der Antifa-Schüler im Laufe des Jahres 1946.

\section{Kader für Deutschland}

Bereits Mitte Mai 1945 war die Umstrukturierung der Antifa-Schulen thematisiert worden. Nach der Abreise der ersten Kader nach Deutschland hatte Wilhelm Pieck vorgeschlagen, die Schulen verstärkt zur Ausbildung von Kadern für die SBZ zu nutzen. Nach den Vorstellungen der KPD sollte dafür im Lager Krasnogorsk ein sogenannter Spezialkurs eingerichtet werden. Gleichzeitig sollten die beiden bisherigen Antifa-Schulen im Lager Juža zusammengelegt, dort allerdings nach wie vor eine unterschiedlich intensive Schulung in einem zweigeteilten Schulsytem durchgeführt werden. Pieck unterschied dabei den Einsatz als Brigadiere in Arbeitslagern („Aktivisten, die als antifaschistische Agitatoren und durch ihr persönliches Beispiel die Umerziehung der Massen der Kriegsgefangenen im Produktionsprozeß fördern") oder als Politinstrukteure, um damit die bisher für das UPVI tätigen KPD-Funktionäre zu ersetzen („Propagandisten, die in den Lagern durch Vorträge, Kurse, Herausgabe von Wandzeitungen, Organisierung des Kulturlebens und durch Mitarbeit an der zentralen Kriegsgefangenenzeitung in der politischen Aufklärungsarbeit und der antifaschistischen Umschulung der Kriegsgefangenen stärker hervortreten" ${ }^{29}$. Die dem OMI Mitte Mai unterbreiteten Vorschläge erläuterte Pieck dem bereits in Deutschland befindlichen Ulbricht, wobei er die Hoffnung auf Kaderzuwachs für die KPD in Deutschland ein wenig dämpfte, weil er sich über die Widerstände des UPVI im Klaren war: „Im wesentlichen sollen sich beide Schulen in 165 [Juža] in ihrem Lehrprogramm einstellen auf die Ausbildung von Kadern für die Arbeit in den Kriegsgefangenenlagern, da doch ein großer Teil der Kriegsgefangenen längere Zeit zur Arbeit in der SU bleiben wird und hier mit dem Wesen der sozialistischen Arbeit und der Sowjetunion aufs engste vertraut gemacht werden müssen und gleichzeitig im Prozeß der Produktionsarbeit ihre antifaschistische Umschulung erhalten. Natürlich sollen aus beiden Kursen der Schule und auch aus den Lagern die geeigneten Kader für die Vorbereitung auf die Arbeit im Lande genommen werden, die zu einer Spezialschule geschickt werden, die an Stelle der jetzigen Schule 40 [Krasnogorsk] treten soll. Du weißt, daß die bisherige Schwierigkeit darin liegt, daß auf dieser Schule das Lehrprogramm an dem Widerspruch leidet, der Ausbildung für die Arbeit in den Kriegsgefangenenlagern und der Arbeit für das Land, wobei die aktuellen Probleme für das Land sehr unzulänglich behandelt werden. Die unterschiedliche Verwendung der Kursanten macht natürlich auch eine unterschiedliche Ausbildung notwendig." 30

28 Ende 1946 war der Aufbau der Antifa-Aktivs beendet; Robel, Antifa, S. $116 \mathrm{ff}$.

29 „Vorschläge für eine Reorganisation der deutschen Kriegsgefangenenschulen“, SAPMOBArch NY 4036/582, Bl. 200-202; als Anlage zu dem Schreiben Piecks an Dimitrov vom 15. 5. 1945, ebenda NY 4036/544, Bl. 192.

30 Pieck an Ulbricht am 22. 5. 1945, in: „Gruppe Ulbricht“, S. 370. 
Den aus Sicht der KPD plausiblen Vorschlägen stand das Interesse des UPVI entgegen, das die Absolventen der Antifa-Schule als Lagerpropagandisten einsetzen wollte. Zudem war das UPVI nicht gewillt, die in den Schulen als Lehrer tätigen Exilkommunisten nach Deutschland zurückkehren zu lassen, denn damit wäre schlagartig fast der gesamte Lehrkörper an den Schulen weggefallen ${ }^{31}$. Die Vorschläge der KPD zur Reorganisation der Schulen standen somit von vornherein im Widerspruch zu den Plänen des UPVI, worauf Pieck Dimitrov hinwies: „Diese Notwendigkeit [unterschiedlicher Schulung] hat bereits in der höheren Kriegsgefangenenschule in Krasnogorsk zu gewissen Schwierigkeiten bei der Gestaltung des Lehrplanes geführt. Der Leiter dieser Schule, Genosse Parfinow [Parfionov], legt in stärkerem Maße Gewicht auf die Ausbildung von Kadern für die Arbeit in den Kriegsgefangenenlagern und demzufolge weniger Gewicht auf die Behandlung der aktuellen Probleme in Deutschland." 32 Noch im Mai warb Pieck im OMI für die Reorganisation der Antifa-Schulen ${ }^{33}$. Mitte August 1945 wiederholte das Auslandsbüro der KPD (Försterling) die Vorschläge zur Umbildung der Antifa-Schulen: „a) Aufgabe der Schule in Krasnogorsk ist es, Funktionäre für die Massenarbeit im Lande zu schulen, die durch die Partei auf den verschiedensten Gebieten des öffentlichen Lebens (Gewerkschaften, Massenorganisationen, Jugendbewegung, Kontrolle des Wirtschaftslebens, Erziehungswesen, Tätigkeit im Magistrat usw.) eingesetzt werden können. b) Die besten Schüler aus Krasnogorsk, die für die Arbeit von Bezirksparteifunktionären und Redakteuren in Frage kommen, müssen wie bisher im Objekt Nr. 12 geschult werden. c) (...) In ihrer Masse sind die Absolventen der Schule [Juža] in den Lagern der Kriegsgefangenen als Leiter der Produktionsarbeit, als Propagandisten und Agitatoren einzusetzen. d) Es wird notwendig sein, unter den Massen von Kriegsgefangenen in den größeren Lagern lokale politische Schulen einzurichten." 34

Trotz der Bemühungen der KPD wurde die Antifa-Schulung nicht umstrukturiert. Es gab auch keine Unterstützung durch das Institut 99. Nach Kriegsende wurde der Lehrplan zwar ein wenig umgestellt, blieb aber hinsichtlich einer Vorbereitung auf Aufgaben in Deutschland nach wie vor sehr vage. Das Programm der um die Jahreswende 1945/46 beginnenden Antifa-Kurse wurde dann zwar geändert, aber es berücksichtigte immer noch nicht die praktischen Anforderungen an einen Einsatz in Deutschland. Im Gegenteil, der Schwerpunkt wurde durch die Einführung des Faches Marxismus-Leninismus auf eine mehr theoretische Schulung verlagert. Die einzige Chance für die KPD, ihre Kaderwünsche zu realisieren, bestand somit in der Fortführung der Parteischulung. Doch durch die Schließung der Schule Nr. 12 im Dezember 1945 wurde ihr auch diese Möglichkeit genommen.

Der Interessenkonflikt um die Fortsetzung der Kader-Schulung spitzte sich zu. Nach dem Abschluß der ersten Nachkriegskurse mit dem leicht veränderten Antifa-Programm kamen Mitte Mai 1946 die Lehrer der Schulen in Krasnogorsk

31 Vgl. Erler, „Moskau-Kader“, S. 255.

32 Pieck an Dimitrov am 15. 5. 1945, SAPMO-BArch NY 4036/544, Bl. 192.

33 Vgl. Notizen Piecks über die Besprechungen bei Dimitrov am 25. 5. und 30. 5. 1945, ebenda, S. 387 und S. 437.

34 Schreiben Försterlings vom 17. 8. 1945. Ein Adressat ist nicht genannt, kann aber nach dem bisher Dargestellten nur Dimitrov gewesen sein, SAPMO-BArch DY 30/IV 2/11/197, Bl. 7+RS. 
zusammen. Auf dieser Versammlung mußte sich die Führung des Instituts 99 schwere Vorwürfe anhören. Heftig kritisierte der Leiter des deutschen Schulungssektors, Wilhelm Zaisser, die fehlende Initiative der Institutsleitung, die trotz der evidenten Mängel des gesamten Unterrichtsprogramms nichts unternehme. Er beklagte, daß durch die schwankende sowjetische Haltung die gesamte Zielsetzung der Antifa-Schulung nicht mehr zu erkennen sei. Es fehle der Bezug zu der politischen Entwicklung in Deutschland: „Wen bilden wir aus? Anfangs hieß es, daß wir Kader für die deutsche KP ausbildeten, dann sagte man uns, daß es Kader für die Arbeit in den Lagern seien. Die Leitung des Instituts ist nicht zu erkennen. Zum Beispiel gab es die politische Situation, daß sich KPD und SPD vereinigten. Wir wußten nicht, ob wir diese Frage thematisieren sollten. Genosse Parfionov [der Schulleiter] sagte, solange es keine Erläuterungen gebe, solle diese Frage nicht zum Thema gemacht werden. Ich denke, daß das richtig war. Er sagte, daß ich Anweisung vom Genossen Černikov [Institut 99] bekommen werde. Aber diese Anweisung ist bis heute nicht eingetroffen." 35 Auch die übrigen Lehrer - des deutschen wie des rumänischen Sektors - beklagten, daß ihnen die Institutsleitung nichts mitteile und sie daher nicht wüßten, worauf sie die Schüler eigentlich vorbereiten sollten ${ }^{36}$. Černikov, der Leiter der Unterrichtsabteilung im Institut 99, erwiderte auf die "gerechtfertigten Vorwürfe“, daß man nicht wisse, „ob wir für den Einsatz im Lager oder im Land ausbilden“. Er schloß mit dem Eingeständnis: „Es gab keine programmatische und methodische Anleitung. Das ist richtig, darüber gibt es nichts weiter zu sagen. " ${ }^{77}$ Diese Einlassung, der sich der Leiter des Instituts (Romanov) im Schlußwort der Versammlung anschloß, macht deutlich, daß es im Mai 1946 von Seiten des Instituts 99 keine klare Zielsetzung bei der Schulung von Kriegsgefangenen gab.

Die passive Haltung des Instituts 99 war insofern erstaunlich, als sich die Politische Hauptverwaltung noch Ende März 1946 sehr zufrieden über den Einsatz der Kriegsgefangenen in Deutschland geäußert hatte. Šikin - nun Leiter des GlavPURKKA ${ }^{38}$ - forderte von der Propagandaabteilung des ZK der VKP (b) (Aleksandrov) sogar mehr Antifa-Kader an: „Angesichts der guten Erfahrungen mit der Arbeit deutscher antifaschistischer Kriegsgefangener, die von uns in Deutschland eingesetzt wurden, ist es unumgänglich zusätzlich 2000 deutsche Kriegsgefangene aus den Reihen derjenigen, die die antifaschistische Schule und Kurse in der UdSSR absolviert haben, der Verwaltung der SMAD für politische Kontrolle zur Verfügung zu stellen, um sie in den Selbstverwaltungsorganen und in demokratischen Organisationen zu verwenden. " 39 Šikins Forderung nach dem vermehrten Einsatz von Antifa-Schülern in der SBZ stand in direktem Zusammenhang mit der

35 Beitrag Wilhelm Zaissers (Leiter des deutschen Sektors) auf der Sitzung; „Protokoll über die Versammlung der Lehrer der Politschule am 13./14. 5. 1946“, MMNA 1463/15 (= RGVA/K 4p/4/36, Bl. 226).

36 Vgl. die Wortbeiträge von Zöllner, Dürr und Spiru (rumänischer Sektorleiter), ebenda (= B1. 230-233).

37 Ebenda (= B1. 234).

38 Ščerbakov war am 10. 5. 1945 gestorben.

39 „Bericht des Leiters des GlavPURKKA an den Leiter der Abteilung für Propaganda und Agitation des ZK der VKP (b) Aleksandrov über die Ergebnisse der Überprüfung der Propagandaverwaltung der SMAD“ vom 30. März 1946, ebenda, S. 147. 
Tätigkeit der Propagandaverwaltung der SMAD. Diese Abteilung war im Oktober 1945 gegründet worden, nachdem deutlich geworden war, daß durch die Zulassung von Parteien das politische Leben in der SBZ mit dem bestehenden Apparat der SMAD nicht zu kontrollieren war ${ }^{40}$. Die Mitarbeiter der 7. Verwaltung des GlavPURKKA, die tragende Säule beim Aufbau der neuen Abteilung, knüpften dabei an ihre Erfahrungen mit antifaschistisch geschulten Kriegsgefangenen in der Frontpropaganda bzw. beim Aufbau der Kommunalverwaltung in der SBZ an, insbesondere der Leiter der Propagandaverwaltung, Oberst Tjul'panov. So war es nur konsequent, daß die Politische Hauptverwaltung im Frühjahr 1946, nachdem der Aufbau der Propagandaverwaltung abgeschlossen war, auf diese Erfahrungen zurückgriff.

Der einzige unmittelbare Nutznießer bei der Verwendung von Antifa-Schülern in der SBZ war aber nicht die Propagandaverwaltung, sondern - so weit es sich nachweisen läßt - der Sicherheitsapparat der SMAD. Dessen Leitung lag in der Hand des NKVD-Generals Serovs, der zugleich stellvertretender Chef der SMAD für Zivilangelegenheiten war. Parallel zu den Ortskommandanturen hatte das NKVD ein flächendeckendes Netz sogenannter operativer Sektoren und Gruppen aufgebaut, die unter anderem maßgeblich an der Verhaftung und Internierung von NS-Funktionsträgern beteiligt waren ${ }^{41}$. Im Juni 1946 schlug das Institut 99 eine Gruppe von 481 Antifa-Schülern zur Repatriierung vor ${ }^{42}$, von denen 257 im August nach Deutschland geschickt wurden. Dort standen sie dem operativen Sektor Berlin (Generalmajor Aleksej Sidnev) ${ }^{43}$ zur Verfügung. Erst im nachhinein wurde die SED durch die Propagandaverwaltung der SMAD von der Repatriierung der Gruppe informiert. Sie hatte jedoch keinen Zugriff auf die Kader. In einem Gespräch mit Tjul'panov am 19. September notierte sich Wilhelm Pieck: „20. 8. Gruppe Kriegsgef.[angener] aus Juscha [Juža] - 257 - General Sietjew soll hier sein - zu dessen Verfügung." 44

Im Spätsommer 1946 machte die Propagandaverwaltung der SMAD noch einen zweiten Versuch, Antifa-Schüler für die Arbeit in der SBZ zu bekommen. Dabei ging es nicht mehr um den Aufbau der Kommunalverwaltungen, sondern um die bevorstehenden Wahlen in der SBZ. Den Antifa-Schülern wurde nun die Aufgabe von Wahlhelfern für die SED zugedacht. Dieser Funktionswandel stand im $\mathrm{Zu}$ sammenhang mit der Arbeitsweise der Propagandaverwaltung, die sich seit dem Zusammenschluß der beiden Arbeiterparteien im April 1946 fast ausschließlich auf den gesellschaftspolitischen Einfluß der SED verließ. So hieß es in dem Bericht der 7. Verwaltung des GlavPURKKA vom 2. September 1946: „Zur Gewährleistung des Sieges der SED bei den Wahlen in Berlin ist es notwendig, sofort alle Maßnah-

40 Zur Propagandaverwaltung siehe Creuzberger, Die sowjetische Besatzungsmacht, S. 35 ff.; Foitzik, Sowjetische Militäradministration, S. $143 \mathrm{ff}$.; Bonwetsch, in: Sowjetische Politik in der SBZ, S. XXVIIff.

41 Foitzik, Sowjetische Militäradministration, S. $161 \mathrm{ff}$.; Petrov, Die Apparate des NKVD/ MVD, S. $143 \mathrm{ff}$;; Sacharov/Fillippovych/Kubina, Tschekisten in Deutschland, insbes. S. $301 \mathrm{ff}$.

42 Romanov an Suslov am 18. 6. 1946 und 27.6. 1946, RGASPI 17/128/58, Bl. 16 u. Bl. 32.

43 Foitzik, Sowjetische Militäradministration, S. 452 u. 477.

44 Notizen Piecks über eine Besprechung bei Tjul'panov am 19.9. 1946, in: Wilhelm Pieck Aufzeichnungen zur Deutschlandpolitik 1945-1953, S. 81. 
men zur Beseitigung der erwähnten Mängel zu ergreifen und schon jetzt alle Aufmerksamkeit der Propagandaverwaltung und des Parteivorstandes der SED auf Berlin zu lenken. Außerdem ist folgendes nötig: (...) 2. Die Frage der Entsendung von 200 überprüften Antifaschisten zur Arbeit unter der deutschen Intelligenz sowohl in Berlin als auch in der Provinz muß dringend entschieden werden. " 45 Aber die Politik der Propagandaverwaltung war in die Kritik geraten. Auf Veranlassung von Ždanov und Kuznecov wurde eine Überprüfungskommission eingesetzt ${ }^{46}$. Die näheren Zusammenhänge zwischen der Krise der Propagandaverwaltung und dem Einsatz von Antifa-Kadern in der SBZ können leider nicht belegt werden, aber zu dem Einsatz von Kriegsgefangenen als Wahlhelfer der SED ist es nicht gekommen, obwohl das offensichtlich von der sowjetischen Führung geprüft wurde.

Anfang Juni 1946 berichtete das OMI über die Schulungstätigkeit des Instituts 99 an eben jene Funktionäre, die die Überprüfung der Propagandaverwaltung der SMAD veranlaßt hatten (Ždanov und Kuznecov). Es sprach sich für eine Fortsetzung des Kadereinsatzes aus: „Nach dem Krieg sind die Antifaschisten, die in ihre Heimatländer geschickt wurden, teilweise von der sowjetischen Militärkommandantur verwendet worden, und die Mehrzahl von ihnen ist in demokratischen Organisationen aktiv. Von ihnen wurden viele Mitglieder kommunistischer Parteien. (...) Die Führungen der kommunistischen Partei Deutschlands, Österreichs und Ungarns bitten darum, die Absolventen der antifaschistischen Schule [Krasnogorsk] und der Kurse [Juža] in ihre Heimatländer zu schicken. Diese Gesuche werden auch von den sowjetischen Militäradministrationen befürwortet. Wir meinen, daß die Vorbereitung von kriegsgefangenen Antifaschisten auf die Arbeit in den entsprechenden Ländern auch in Zukunft fortgesetzt werden sollte. " 47 Aber trotz der Befürwortung durch das OMI wurde der Kadereinsatz von Kriegsgefangenen gestoppt. Im Sommer 1946 kam es zu einem grundlegenden Wandel in der sowjetischen Bewertung des Einsatzes von Antifa-Schülern in der SBZ.

\section{Reorganisation der Antifa-Schulen}

Seit der Auflösung des Nationalkomitees wurden Antifa-Schüler nicht mehr zum Nutzen der KPD/SED in Deutschland eingesetzt. An dieser Entwicklung waren die deutschen Kommunisten nicht ganz unbeteiligt. Unmittelbar nach der Auflösung des NKFD hatte Weinert an die Parteileitung der KPD appelliert, daß von Deutschland aus „noch einmal mit allem Nachdruck auf die Sorge um diese Kader“ hingewiesen werden solle ${ }^{48}$. Nachdem er nach Deutschland zurückgekehrt war, mußte er

45 „Bericht des Mitarbeiters der 7. Verwaltung der politischen Hauptverwaltung Oberstleutnant Konstantinovskij über den Verlauf der Vorbereitungen auf die Stadt- und Bezirksverordnetenwahlen in Berlin" vom 2. 9. 1946, in: Sowjetische Politik in der SBZ 1945-1949, S. 68; vgl. Naimark, Russen in Deutschland, S. 415; Creuzberger, Die sowjetische Besatzungsmacht, S. 91.

46 Siehe Naimark, Russen in Deutschland, S. $410 \mathrm{ff}$.; Bonwetsch, in: Sowjetische Politik in der SBZ, S. XXXII ff.; Dokumente, ebenda, S. $221 \mathrm{ff}$.

47 Suslov an Ždanov und Kuznecov am 5. 6. 1946, RCChDINI 17/128/846, Bl. 66-67.

48 Weinert an Pieck am 3. 11. 1945, SAPMO-BArch NY 4065/5, Bl. 43. 
jedoch feststellen, daß seitens der Partei nichts unternommen worden war49. Zwar beschloß das Zentralsekretariat der SED auf seiner Sitzung am 4. Mai 1946, wegen der bevorzugten Entlassung antifaschistisch geschulter Kriegsgefangener „Besprechungen aufzunehmen" 50 , aber schon Ende Mai kam die ernüchternde Antwort: „Eine nochmalige Anfrage bei der SMA Karlshorst [SMAD] wurde dahingehend beantwortet, daß es bei der SMA keine Stelle gibt, die eine bevorzugte Entlassung aus russischer Kriegsgefangenenschaft bearbeitet. Anträge und Anfragen sind daher zwecklos. Antifaschisten kommen im Rahmen der allgemeinen Entlassungen nach Hause." 51

Das Interesse der SED an „antifaschistischen Kriegsgefangenen“ aus der Sowjetunion bezog sich auf einen eng gefaßten Personenkreis. Allein der Abschluß der Antifa-Schule war für die Kaderverwaltung der SED (Personalpolitische Abteilung - PPA) keine ausreichende Qualifikation. Ihre Aufmerksamkeit galt nahezu ausschließlich der Repatriierung von Parteimitgliedern, bevorzugt Parteifunktionären mit Organisationserfahrung. Lediglich für diese Gefangenen setzte sich die PPA ein. Nach der abschlägigen Auskunft der SMAD im Frühjahr 1946 konzentrierte sich die SED auf die Repatriierung folgender Kategorien von Kriegsgefangenen: (1) „Frühere Funktionäre der Partei, die von der Parteiorganisation zur Parteiarbeit angefordert wurden“, (2) „Ehemalige Mitglieder der Partei und Funktionäre von proletarischen Massenorganisationen“, (3) „Söhne von Funktionären, Parteimitgliedern sowie Antifaschisten, für welche die Parteiorganisationen die politische Bürgschaft über ihre Zuverlässigkeit übernehmen." 52 Abgesehen von den sehr spezifischen Kadervorstellungen der SED bestand ihr größtes Problem darin, daß sie ihre Wünsche bei der SMAD anmelden sollte ${ }^{53}$, diese sich aber nicht kooperativ zeigte. Es war für die SED nicht möglich, über das immer noch in Moskau existierende Auslandsbüro der KPD (Försterling) entsprechende Kaderanforderungen zu stellen. Und da die Verbindung nach Moskau - abgesehen von persönlichen Spitzengesprächen - nicht funktionierte, war die SED ohne jeglichen Einfluß auf die Repatriierung von kriegsgefangenen Kadern. Darüber entschieden allein das OMI bzw. das Institut 99 in Moskau. Im Sommer 1946 ergab sich damit die Konstellation, daß sich das OMI für die Repatriierung von Antifa-Kadern zur Unterstützung der SED aussprach, diese Kader tatsächlich aber nur für die SMAD verwendet wurden. SMAD und OMI handelten nach eigenem Gutdünken und ohne auf die Bedürfnisse der SED einzugehen, vor allem ohne die Verfügungsgewalt über die eingesetzten Antifa-Kader abzugeben. Man konnte den Eindruck gewinnen - so Bonwetsch -, „daß sich die Vertreter der Propagandaverwaltung für die Aktivitäten der SED verantwortlicher fühlten als deren eigene Funktionäre."54

49 Weinert an das ZK der KPD am 1. 4. 1946, SAPMO-BArch DY 30/IV 2/11/196, Bl. 28.

50 SAPMO-BArch DY 30/IV 2/2.1/4, B1. 3.

51 Aktenvermerk der Personalpolitischen Abteilung der SED vom 31. 5. 1946, SAPMO-BArch NY 4182/1191, Bl. 135.

52 „Zur Frage der Entlassung antifaschistischer Kriegsgefangener“ vom 13. 7. 1946, SAPMOBArch DY 30/IV 2/11/196, Bl. 46.

53 Vgl. Berija an Molotov am 22. 12. 1945, GARF 9401/2/105, Bl. 385; Kruglov an Molotov am 5. 4. 1946, ebenda Akte 142, Bl. 168.

54 Bonwetsch in der Einleitung zu: Sowjetische Politik in der SBZ, S. XLIV; vgl. Creuzberger, Die sowjetische Besatzungsmacht, S. $57 \mathrm{ff}$. 
Ebenso wie OMI und SMAD machte sich das UPVI ungefragt zum Fürsprecher der SED. Im Juni 1946 brachte die operative Abteilung des UPVI den Plan ins Gespräch, anstelle des aufgelösten Nationalkomitees einen SED-Soldatenbund zu schaffen, der als Nachfolgeorganisation des NKFD auftreten solle. Kobulov, seit Anfang 1945 Leiter der operativen Abteilung des UPVI, berichtete am 12. Juni 1946 Innenminister Kruglov 55 , Generalfeldmarschall Paulus habe sich bereit erklärt, nach den Vorgaben des UPVI einen entsprechenden Vorschlag auszuarbeiten ${ }^{56}$. Vorgesehen war die Schaffung eines Soldatenbundes als reguläre Parteiorganisation der SED. Da das offensichtlich auf Kritik stieß, wurde wenige Tage später eine überarbeitete Fassung vorgelegt. Aber immer noch waren die Organisationsprinzipien einer kommunistischen Kaderpartei bestimmend. Der zweite Entwurf, tituliert mit „Die SED und die Kriegsgefangenen in der Sowjetunion“, unterschied zwischen „Anhängern“, d.h. Mitgliedern, und „Sympathisanten“, d.h. Kandidaten des Soldatenbundes. Der Gedanke einer Parteienpluralität wurde mit dem Hinweis auf die „führende Rolle der SED“ verworfen: „Eine derartige Lösung der Frage könnte Erinnerungen an die bereits überholte Bewegung des Nationalkomitees aus dem Jahre 1943 wachrufen. Insbesondere könnten erneut verschwommene Vorstellungen über Parität [paritätische Gremienbesetzung] und Stimmgleichheit aufkommen, obwohl heutzutage nur die Rede von einer zeitweiligen Taktik der Zusammenarbeit unter der Führung der SED sein kann. " ${ }^{77}$ Gegenüber dem ZK der VKP (b) trat das dem UPVI vorgesetzte Innenministerium allerdings zurückhaltender auf. In dem Bericht Kruglovs an Ždanov vom 15. Juni 1946 ist lediglich von einem „Demokratischen Bund deutscher Kriegsgefangener in der Sowjetunion" die Rede, der zur „Stärkung und Vereinigung antifaschistischer Elemente unter den Kriegsgefangenen, die sich der [Politik der] Sowjetunion und der Sozialistischen Einheitspartei Deutschlands anschließen, und gleichzeitig zur Eindämmung faschistischer Propaganda" dienen solle. Die Organisationsprinzipien entsprachen exakt dem Vorgehen bei Gründung des NKFD: Bildung einer Initiativgruppe (unter Leitung von Paulus), Ausarbeitung eines Statuts und Gründungskonferenz ${ }^{58}$.

Der Vorstoß des UPVI läßt die Vermutung aufkommen, daß es möglicherweise nie mit dem Konzept des NKFD konform gegangen war und sich nur der Politik des ZK der VKP (b) (Manuil'skij, Dimitrov) gebeugt hatte. Bereits im August 1944 hatte das UPVI versucht, den Generalfeldmarschall als Leitfigur eines „Gegenkomitees" aufzubauen, war aber vom Institut 99 gestoppt worden ${ }^{59}$. So stützte sich das UPVI auf Paulus erst wieder zu einem Zeitpunkt, zu dem sich die politische Situation in der SBZ und die personellen Entscheidungsstrukturen in Moskau entscheidend gewandelt hatten. Im April 1946 war das Parteiensystem in der SBZ durch die Schaffung der SED grundlegend verändert worden. Gleichzeitig wurde das OMI,

55 Kruglov löste Berija am 30. 12. 1945 in der Leitung des NKVD ab, das Mitte März 1946 in Ministerium für Innere Angelegenheiten (MVD) umbenannt wurde; Lubjanka, S. $46 \mathrm{ff}$.

56 Kobulov an Kruglov am 12. 6. 1946, in: Rešin, „Bol’šaja čast' voennoplennych“, S. 103 f.; vgl. ders., Feldmarschall im Kreuzverhör, S. $186 \mathrm{ff}$. Im folgenden wird aus der russischen Veröffentlichung Rešins zitiert, die im Vergleich zu der deutschen Publikation, die wissenschaftlichen Standards überhaupt nicht entspricht, wenigstens etwas exakter ist.

57 Ebenda, S. 104.

58 Kruglov an Ždanov am 15. 6. 1946, ebenda, S. 107.

59 Siehe oben, S. $88 \mathrm{f}$. 
nachdem Dimitrov bereits im Dezember 1945 nach Bulgarien zurückgekehrt war, reorganisiert ${ }^{60}$. War die Auflösung des Nationalkomitees noch mit der Schaffung des Blockes antifaschistischer Parteien in der SBZ begründet worden, so ergab sich nach der Vereinigung von KPD und SPD eine deutliche Hegemonialstellung der SED. Unverblümt hieß es daher in dem Vorschlag zur Gründung des SED-Soldatenbundes: „Die Hauptaufgabe besteht darin, unter den Kriegsgefangenen eine möglichst große Anzahl überzeugter und überprüfter ,Anhänger' sowie Sympathisanten zu gewinnen und zu vereinen. Sie müssen bereit und zugleich in der Lage sein, zukünftig in der Heimat nach den Prinzipien und Zielen der SED an der Stelle, an die sie beordert werden, zu arbeiten." 61

Das OMI zeigte sich mit der Initiierung einer neuen Bewegung prinzipiell einverstanden, da seit der Auflösung des Nationalkomitees die politische Arbeit unter den deutschen Kriegsgefangenen „erheblich schwächer" geworden sei. Jedoch kritisierte der neue OMI-Chef Suslov in seiner Antwort vom 4. Juli die Nominierung von Paulus, "weil das im Ausland als Maßnahme zur Gründung einer deutschen Armee aus Kriegsgefangenen auf dem Territorium der Sowjetunion ausgelegt werden kann". Um dieser Kritik vorzubeugen, schlug er vor, den Soldatenbund als „innere Maßnahme“ ohne öffentliche Erwähnung in der sowjetischen Presse zu gründen ${ }^{62}$. Das OMI bevorzugte damit ein Vorgehen, wie es Ende 1944 bereits mit dem Antifaschistischen Büro österreichischer Kriegsgefangener praktiziert worden war. Der mit diesem Vorschlag verbundene Hintergedanke war, einen unmittelbaren deutschlandpolitischen Bezug zu vermeiden. Das österreichische Antifa-Büro war für die sowjetische Außenpolitik nie funktionalisiert worden.

Das Projekt eines SED-Soldatenbundes scheiterte. Die außenpolitischen Bedenken, die das OMI äußerte, waren dabei sicherlich ein ernst zu nehmender Einwand. Gerade im Sommer 1946 kursierten in der britischen Presse Gerüchte über eine „Paulus-Armee"63. Außenpolitischem Schaden hätte man aber - das OMI hatte es selbst vorgeschlagen - dadurch aus dem Weg können, daß der Soldatenbund als „innere Maßnahme" des UPVI organisiert worden wäre. Entscheidender war, daß das OMI das Interesse an der Verwendung von Kriegsgefangenen als Kader in der SBZ verloren hatte. War schon die Antifa-Schulung nicht entsprechend den Wünschen der KPD umgestellt worden, so war auch die Initiierung eines SED-Soldatenbundes nur sinnvoll, wenn man dessen Mitglieder auch in der SBZ einsetzen wollte. Statt dessen schlug Suslov vor, die gesamte politische Arbeit dem Verantwortungsbereich des UPVI zu unterstellen: „Zieht man in Betracht, daß sich in den Lagern des MVD in der Sowjetunion gegenwärtig über zwei Millionen Kriegsgefangene befinden, und daß eine erzieherische Arbeit unter diesen Kriegsgefangenen unerläßlich ist, wäre es notwendig, durch eine Entscheidung des ZK das MVD zur Durchführung einer systematischen politisch-erzieherischen und kulturell-aufklärerischen Arbeit unter den Kriegsgefangenen zu verpflichten." 64

60 Adibekov, Kominform, S. $14 \mathrm{f}$.

61 „Die SED und die Kriegsgefangenen in der Sowjetunion“, in: Rešin, „Bol’šaja čast’ voennoplennych", S. 105.

62 Suslov an Ždanov am 4. 7. 1946, RGASPI 17/128/846, Bl. 93.

63 Bungert, Das Nationalkomitee und der Westen, S. 277.

64 Suslov an Ždanov am 4. 7. 1946, RGASPI 17/128/846, Bl. 94. 
Im September 1946 wurde schließlich im Zuge einer allgemeinen Reorganisation des OMI die Auflösung des Instituts 99 zum 31. Dezember 1946 beschlossen. Die Schließung war vor dem Hintergrund dauernder Kompetenzstreitigkeiten zwischen Institut 99 und UPVI eine plausible Lösung ${ }^{65}$. Aber das späte Einlenken des OMI zeigt, daß bis zum Spätsommer 1946 alle Möglichkeiten offengehalten werden sollten. Erst im Juni 1946 wurde dem UPVI per ZK-Beschluß wieder eine eigenständige Politabteilung zugestanden. Und der für die Umsetzung dieser Entscheidung notwendige MVD-Befehl erging sogar erst im Oktober 1946, nachdem die Auflösung des Instituts 99 zum 31. Dezember beschlossen worden war. Der Aufbau der Politabteilungen in den Lagern sollte bis zum 1. Januar 1947 abgeschlossen sein ${ }^{66}$. Eine weitere Verfügung des MVD regelte im Dezember 1946 die Übernahme der Antifa-Schulen, der Redaktionen der deutschen, ungarischen, rumänischen und österreichischen Kriegsgefangenenzeitungen und der Registratur der Antifa-Kader. Dafür wurde der Politabteilung des UPVI eine eigenständige Abteilung für die antifaschistische Arbeit unter den Kriegsgefangenen (mit 55 Mitarbeitern) beigeordnet. Dieser wurden die Politinstrukteure in den Lagern, die immer ausschließlich dem UPVI und nie dem Institut 99 unterstanden hatten, unterstellt ${ }^{67}$. Nun erst war das UPVI alleinverantwortlich für die gesamte politische Arbeit unter den Kriegsgefangenen ${ }^{68}$. Unter der Regie der neuen Abteilung wurde das System der AntifaSchulen erheblich ausgebaut. $\mathrm{Zu}$ den bisherigen beiden Schulen in Krasnogorsk und Juža (nun sogenannte Zentrale Antifa-Schulen) kamen ab 1947 etwa 50 Gebietsund ca. 120 Lagerschulen hinzu. Alle Schulen bildeten in unterschiedlich intensiven Lehrgängen Propagandisten aus, die die Antifa organisieren, insbesondere aber als Aktivisten die Leistung der Arbeitsbrigaden in den Kriegsgefangenenlagern steigern sollten ${ }^{69}$.

Anfang 1947 war das Interesse des ZK der VKP (b) an einem Einsatz politisch geschulter Kriegsgefangener in der SBZ erlahmt. Das bekam auch die Führung der SED zu spüren, die Ende Januar 1947 nach Moskau reiste. Auf die Bitte der SED, sich doch intensiver um die Antifa-Kader in den Kriegsgefangenenlagern zu kümmern, wurde lapidar auf fehlendes Personal des UPVI - viele Emigranten, die als Politinstrukteure gearbeitet hatten, waren in ihre Heimatländer zurückgekehrt - und auf Arbeitsüberlastung des ZK verwiesen. So blieb der SED nicht anderes übrig, als um eine Fortführung der politischen Arbeit unter den Kriegsgefangenen unter Ver-

$65 \mathrm{Vgl}$. Adibekov, Kominform, S. 11. Adibekov motiviert den ZK-Beschluß mit der doppelten Verwaltungszuständigkeit von UPVI und Institut 99, die sich negativ auf die Arbeit der Antifa-Schulen ausgewirkt habe. Dieses an sich plausible Argument war aber nicht der tatsächliche Grund. Den Ausschlag gab die veränderte Haltung des OMI.

${ }^{66}$ MVD-Befehl Nr. 00933 vom 19. 10. 1946 zur Ausführung des ZK-Beschlusses vom 26.6. 1946, RGVA/K 1p/37a/2, Bl. 139-142 RS, ediert in: Voennoplennye v SSSR, S. 133; siehe auch ebenda, S. 925, Anm. 1 zu Dok. 2.2.

67 Anlage zum MVD-Befehl Nr.001116-1946 vom 9. 12. 1946, RGVA/K 1p/37a/2, Bl. 143-145. Die Anlage trägt kein Datum; zur Zuordnung siehe Anm. 1 zu Dok. 2.28, in: Voennoplennye v SSSR, S. 930.

$68 \mathrm{Vgl}$. Libera, Zur Entwicklung der antifaschistischen Bewegung, S. 49. Libera beschreibt den Aufbau der Politabteilung bzw. der ihr zugeordneten Abteilung für die antifaschistische Arbeit unter den Kriegsgefangenen zutreffend, datiert ihn aber irrtümlich auf den 1. Januar 1946.

69 Ebenda, S. 158f.; Robel, Antifa, S. 202. 
wendung der Absolventen der Antifa-Schulen zu bitten. Damit jedoch gab die SED das Ziel auf, Antifa-Schüler als Kader nach Deutschland zurückzuholen. Was blieb, war die Bitte um Repatriierung derjenigen kommunistischen Kriegsgefangenen, deren Namen die PPA seit Anfang 1946 gesammelt hatte. Im Protokoll des Gesprächs der SED-Führung bei Stalin wurde vermerkt: „[SED:] (...) Wir wünschten, daß die demokratische Erziehung der Kriegsgefangenen verstärkt würde. [Entgegnung von sowjetischer Seite:] Lassen Sie Deutsche die politische Arbeit durchführen. [SED:] Im Lager befinden sich demokratische Elemente, aber sie werden nicht in gebührendem Maße genutzt. Wir möchten, daß das ZK der VKP (b) sich mit dieser Frage befaßt. [Entgegnung:] Das ZK der VKP (b) ist zu sehr überlastet. [SED:] Wir bitten darum, daß geschulte antifaschistische Kriegsgefangene die Propaganda unter den Kriegsgefangenen durchführen. Wir bitten ebenfalls darum, daß einige Hundert Antifaschisten laut Liste nach Deutschland geschickt werden. [Entgegnung:] Wir werden sie schicken. " 70 Nur durch persönliche Vorsprache und auf der Grundlage vorbereiteter Namenslisten gelang es der SED, einige Kader für den Einsatz in Deutschland geschickt zu bekommen ${ }^{71}$. Und trotz der Zusicherung durch Stalin und Bestätigung durch das OMI bekam die SED im Sommer 1947 nicht diejenigen, die sie namentlich angefordert hatte. Als im Juni/Juli 1947 ca. 500 „Antifaschisten“ repatriiert wurden, stellte sich heraus, daß nur zur Hälfte die von der SED gwünschten Kriegsgefangenen dabei waren. Die Entlassenen bedeuteten somit nicht den von der SED erhofften Kaderzuwachs ${ }^{72}$.

Im Sommer 1947, anläßlich eines Heimkehrertransportes mit bevorzugt entlassenen Antifa-Schülern, stellte sich in Frankfurt/Oder Oberst Prichodkov als Leiter der politischen Abteilung des UPVI vor ${ }^{73}$. Wie die PPA im Anschluß an dieses Gespräch der SED-Führung berichtete, sei in den „letzten sieben Monaten“, d.h. seit Auflösung des Instituts 99, die politische Arbeit unter den deutschen Kriegsgefangenen in der Sowjetunion verstärkt worden. $\mathrm{Zu}$ Optimismus war dennoch kein Anlaß, denn wie die Nachfragen Prichodkovs erkennen ließen, war die sowjetische Haltung zur Fortsetzung der Antifa-Schulung noch indifferent. Getreulich berichtete die PPA: „Er [Prichodkov] wünscht von uns einen Bericht, wie sich die Obengenannten [antifaschistischen Kriegsgefangenen] bewährten, und ob es sich lohnt, die antifaschistische Schulungsarbeit in der SU weiter durchzuführen. Außerdem machte Oberst Prichodkov den Vorschlag, daß die SED darüber beraten solle, in

70 Bonwetsch/Bordjugov, Stalin und die SBZ, S. 301.

$71 \mathrm{Vgl}$. die drei Listen mit "Namen deutscher Antifaschisten in russischer Kriegsgefangenschaft" vom 4., 6. und 20.12. 1946, SAPMO-BArch NY 4036, Akte 745, Bl. 28-70. Es handelte sich um Parteifunktionäre der KPD („Liste Nr. 1“ mit 26 Namen), Mitglieder der KPD („Liste Nr. 2“mit 93 Namen) und Söhne von KPD-Funktionären („Liste Nr. 3“mit 342 Namen). Die Kategorisierung geht aus der Liste nicht hervor, ist aber wegen der oben (S. 191) geschilderten Erfassungskriterien der PPA anzunehmen.

72 „Rückführung deutscher antifaschistischer Kriegsgefangener aus der SU“, PPA an Pieck am 30. 7. 1947, SAPMO-BArch DY 30/IV 2/11/196, Bl. 66; vgl. Bestätigung der von Stalin zugesicherten Repatriierungen durch das OMI am 20. 2. 1947, ebenda NY 4036/745, B1. 77; siehe auch: Morré, Kader für Deutschland, S. $220 \mathrm{ff}$.

73 Das war nicht ganz zutreffend: Oberst Prichodkov war Leiter der Abteilung für die antifaschistische Arbeit unter den Kriegsgefangenen, Leiter der Politabteilung war General Sobolevskij; Libera, Zur Entwicklung der antifaschistischen Bewegung unter den deutschen Kriegsgefangenen, S. 49, Anm. 78. 
welcher Form die Ost-Heimkehrer organisatorisch erfaßt werden könnten. Er will darüber mit Oberst Tjulpanov [Propagandaverwaltung der SMAD] sowie mit dem Vorstand des Zentralsekretariats [der SED] sprechen."74 Diese Episode bestätigte die pessimistische Einschätzung Wilhelm Zaissers, des ehemaligen Leiters des deutschen Sektors an der Antifa-Schule Krasnogorsk, die dieser im Februar 1947 nach seiner Rückkehr nach Deutschland gegenüber der SED-Führung machte. Angesichts der Auflösung des Instituts 99 warnte er unter Berufung auf den ehemaligen Institutschef Romanov, „daß die bisherige Zielsetzung der Schule, nämlich geeignete antifaschistische Kräfte auszubilden für die Arbeit in Deutschland (die zwar offiziell nicht geändert worden ist), praktisch aber doch illusorisch werden wird. Er [Romanov] erklärte mir wörtlich, daß die Politverwaltung [des UPVI] selbstverständlich offiziell daran festhalten werde, Kräfte für die Arbeit im Lande auszubilden, aber bei Abschluß der Schule unter diesen oder jenen Vorwänden doch den größten Teil der Schüler auf die einzelnen Lager als Aktivisten verteilen würde." Zaisser führte die Haltung des UPVI unter anderem auch auf das fehlende Interesse der SED an der Antifa-Schulung und das schlechte Auftreten Försterlings als deutscher Parteivertreter in Moskau zurück. Die Beziehungen der deutschen Parteileitung in Moskau zum Institut 99 seien "gleich Null“ gewesen und Försterling habe bei den sowjetischen Stellen "nicht die geringste Autorität" genossen ${ }^{75}$. Zaisser schlug vor, die Antifa-Schulung entweder nach Deutschland zu verlegen oder, wenn das nicht möglich sei, den Unterrichtsbetrieb stärker auf russische Politoffiziere zu stützen: „Diese Meinung drängt sich mir heute noch viel mehr auf, da alle Wahrscheinlichkeit dafür spricht, daß das Schwergewicht in der Schule, ob wir wollen oder nicht, mehr auf die Erziehung von Aktivisten für die Gefangenenlager verschoben wird. So wie die Dinge heute liegen, wäre es meiner Ansicht nach zweckmäßiger zu versuchen, die dort [in der Antifa-Schule] noch als Lehrer befindlichen deutschen Genossen nach hier [SBZ] zu holen, da sie hier weitaus zweckmäßiger ausgenutzt werden könnten. Man müßte sich dann hier allerdings klar darüber sein, daß vorläufig auf einen nennenswerten Kräftezuwachs für Deutschland aus den Kreisen der Absolventen der Schule nicht zu rechnen wäre."76

Die Auflösung des Instituts 99 hatte zur Folge, daß bis zu der beginnenden Repatriierung aller deutschen Kriegsgefangenen aus der Sowjetunion an den AntifaSchulen nur noch Kader für das UPVI ausgebildet wurden. Diese wurden zum Ausbau der Antifa in den sowjetischen Kriegsgefangenenlagern, aber nicht zur Unterstützung der SMAD oder der SED in Deutschland verwendet.

74 Bericht der PPA vom 21. 6. 1947, SAPMO-BArch DY 30/IV 2/11, Akte 202, Bl. 11.

75 Diese Kritik war kein Einzelfall, vgl. Erler, „Moskau-Kader“, S. 254, Anm. 177.

76 Zaissers „Bericht über die Antifa-Schule“ vom 8. 2. 1947, SAPMO-BArch NY 4036/582, Bl. 209-212. 